\title{
A Retrospective Filter Trust Region Algorithm for Unconstrained Optimization*
}

\author{
Yue Lu, Zhongwen Chen \\ School of Mathematics Science, Suzhou University, Suzhou, China \\ E-mail: yueyue403@gmail.com,zwchen@suda.edu.cn \\ Received June 8, 2010; revised July 18, 2010; accepted July 21, 2010
}

\begin{abstract}
In this paper, we propose a retrospective filter trust region algorithm for unconstrained optimization, which is based on the framework of the retrospective trust region method and associated with the technique of the multi-dimensional filter. The new algorithm gives a good estimation of trust region radius, relaxes the condition of accepting a trial step for the usual trust region methods. Under reasonable assumptions, we analyze the global convergence of the new method and report the preliminary results of numerical tests. We compare the results with those of the basic trust region algorithm, the filter trust region algorithm and the retrospective trust region algorithm, which shows the effectiveness of the new algorithm.
\end{abstract}

Keywords: Unconstrained Optimization, Retrospective, Trust Region Method, Multi-Dimensional Filter Technique

\section{Introduction}

Consider the following unconstrained optimization problem

$$
\min f(x)
$$

where $x \in R^{n}, f: R^{n} \rightarrow R$ is a twice continuously differentiable function.

The trust region method for unconstrained optimization is first presented by Powell [1], which, in some sense, is equivalent to the Levenberg-Marquardt method which is used to solve the least square problems and which was given by Levenberg [2] and Marquardt [3]. The basic idea of trust region methods works as follows. In the neighborhood of the current iterate (which is called the trust region), we define a model function that approximates the objective function in the trust region and compute a trial step within the trust region for which we obtain a sufficient model decrease. Then we compare the achieved reduction in $f(x)$ to the predicted reduction in the model for the trial step. If the ratio of achieved versus predicted reduction is sufficiently positive, we define our next guess to be the trial point. If this ratio is not sufficiently positive, we decrease the trust region radius in order to make the trust region smaller. Otherwise, we may increase it or possibly keep it unchanged.

*This work was supported by Chinese NSF Grant 60873116.
Since the trust region method is of the naturalness, the strong convergence and robustness, it has been concerned by many people, such as Powell $[1,4,5]$, Schultz et al. [6], Sorensen [7], Moŕe [8], Yuan [9] and so on. In recent years, the trust region method has been applied to the optimization problems with equality constraints [10], simple bound constraints [11], convex constraints [12] and so on. Many of convergence results have been obtained, which can be seen in [13].

In Fletcher and Leyffer [14] a new technique for globalizing methods for nonlinear programming (NLP) is presented. The idea is referred to as an NLP filter, motivated by the aim of avoiding the need to choose penalty parameters, and considered the relationship between the objective function and the constraint violation in the view of multi-objective optimization. They make the values of the objective function and the constraint violation to be a pair (which is called the filter), construct a sophisticated filter mechanism by comparing the relationship between the pairs, and control the algorithm to converge to the stationary point of the problem (1). The results of numerical tests show that the filter methods are very effective. Fletcher et al. [14,15], Toint et al. [16], Ulbrich et al. [17], Wächter et al. [18,19] have combined the idea with SQP method, trust region method, interior-point method, line search methods, respectively, and obtained some interesting results about the filter method. 
Fletcher, Leyffer and Toint [20] review the ideas above and mention the application of the filter method in practice. In [14], they study the problem of the following form

$$
\begin{aligned}
& \min f(x), \\
& \text { s.t. } c(x) \leq 0,
\end{aligned}
$$

where $c: R^{n} \rightarrow R^{m}$ is continuously differentiable function. Define the measure of the constraint violation

$$
h(c(x))=\sum_{j=1}^{m} \max \left(0, c_{j}(x)\right) .
$$

We use $\left(f^{(k)}, h^{(k)}\right)$ to denote values of $f(x)$ and $h(c(x))$ evaluated at $x_{k}$. Now, we give the following definitions about the filter methods.

Definition 1.1 A pair $\left(f^{(k)}, h^{(k)}\right)$ obtained on iteration $k$ is said to dominate another pair $\left(f^{(l)}, h^{(l)}\right)$ if and only if

$$
f^{(k)} \leq f^{(l)} \text { and } h^{(k)} \leq h^{(l)} .
$$

Definition 1.2 A filter is a list of pairs $\left(f^{(l)}, h^{(l)}\right)$ such that no pair dominates any other.

We use $F_{k}$ to denote the set of iteration indices $j(j \leq k)$ such that $\left(f^{(j)}, h^{(j)}\right)$ is an entry in the current filter.

Definition 1.3 A pair $\left(f^{(l)}, h^{(l)}\right)$ is said to be acceptable for inclusion in the filter if it is not dominated by any pair in the filter, that is, for any pair $\left(f^{(l)}, h^{(l)}\right) \in$ $F_{k},\left(f^{(k)}, h^{(k)}\right)$ satisfies

$$
f^{(k)} \leq f^{(l)} \text { or } h^{(k)} \leq h^{(l)}
$$

In order to obtain the global convergence of the algorithm, we should make $f, h$ satisfy the sufficient reduction condition, so we strengthen the acceptable rule (2) as

$$
f^{(k)} \leq f^{(l)}-\gamma_{h} h^{(l)} \text { or } h^{(k)} \leq\left(1-\gamma_{h}\right) h^{(l)}
$$

where $\gamma_{h} \in(0,1)$. When $\gamma_{h} \in(0,1)$ is very small, there is negligible difference in practice between (2) and (3).

Definition 1.4 When the pair $\left(f^{(k)}, h^{(k)}\right)$ is added to the list of pairs in the filter, any pairs in the filter that are dominated by the new pair are removed, that is, we remove the pair $\left(f^{(l)}, h^{(l)}\right) \in F_{k}$ which satisfies

$$
f^{(k)}<f^{(l)} \text { and } h^{(k)}<h^{(l)} .
$$

This is called the modification of the filter.

Gould et al. [21] and Miao et al. [22] applies the filter technique to unconstrained optimization, whose characteristic is to relax the condition of accepting a trial step for the usual trust region method, which improves the effectiveness of the algorithm in some sense. The nonmonotonic algorithm also has the algorithm in some sense. The nonmonotonic algorithm also has the characteristic [23,24].

Recently, Bastin et al. [25] presents a retrospective trust region method for unconstrained optimization. Comparing their algorithm with the basic trust region algorithm, the updating way of the trust region radius is different, and the retrospective ratio

$$
\begin{aligned}
\tilde{\rho}_{k+1} & =\frac{f\left(x_{k+1}\right)-f\left(x_{k+1}-s_{k}\right)}{m_{k+1}\left(x_{k+1}\right)-m_{k+1}\left(x_{k+1}-s_{k}\right)} \\
& =\frac{f\left(x_{k}\right)-f\left(x_{k}+s_{k}\right)}{m_{k+1}\left(x_{k}\right)-m_{k+1}\left(x_{k}+s_{k}\right)}
\end{aligned}
$$

is mentioned, where $m_{k}\left(x_{k}+s\right)$ is the approximated quadratic model of the objective function $f(x)$ at $x_{k}$. $s_{k}$ is a solution of the following trust region subproblem

$$
\begin{aligned}
& \min m_{k}\left(x_{k}+s\right), \\
& \text { s.t. }\|S\| \leq \Delta_{k} .
\end{aligned}
$$

$\Delta_{k}$ is the trust region radius at the current iterate point. In the basic trust region algorithm, the ratio $\rho_{k}$

$$
\rho_{k}=\frac{f\left(x_{k}\right)-f\left(x_{k}+s_{k}\right)}{m_{k}\left(x_{k}\right)-m_{k}\left(x_{k}+s_{k}\right)}
$$

plays the following two roles.

1) Determine the trial step to be accepted by the algorithm or not.

2) Adjust the trust region radius correspondingly.

In the retrospective trust region method, the two roles are played by the ratio $\rho$ and $\tilde{\rho}$, respectively. In the basic trust region algorithm, the determination of trust region radius is an important and difficult problem. Sartenaer [26] and Zhang et al. [27] present the self-adaptive trust region methods and give some discussions about the determination of trust region radius. Bastin et al. [25] presents a retrospective trust region method for unconstrained optimization. The retrospective ratio in this method uses the information at the current iterate and the last iterate point, which can give the more effective estimation of trust region radius. Hence, the number of solving trust region subproblem may be decreased, which improves the effectiveness of the method.

In this paper we present a new algorithm for unconstrained optimization, which is based on the framework of the retrospective trust region method [25] and associated with the technique of the multi-dimensional filter $[21,22]$. Under reasonable assumptions, we analyze the global convergence of the new method and report the preliminary results of numerical tests. We compare the results with those of the basic trust region algorithm, the filter trust region algorithm and the retrospective trust region algorithm, which shows the effectiveness of the 
new algorithm. This paper is organized as follows. The new algorithm is described in Section 2. Basic assumptions and some lemmas are given in Section 3. The analysis of the first order and second order convergence is given in Section 4 and Section 5, respectively. Section 6 reports the numerical results. Finally, we give some final remarks on this approach.

\section{Algorithm}

In this paper, we define $g(x)=\nabla_{x} f(x)$. At the current iterate $x_{k}, f_{k}=f\left(x_{k}\right), g_{k}=g\left(x_{k}\right), g_{k i}$ denotes the ith component of the vector $g_{k}$. Throughout this paper, $\|\cdot\|$ denotes the Euclidean norm. Now, we review the basictrust region algorithm as follows.

\subsection{Algorithm BTR (Basic Trust Region Algorithm)}

Step 0. (Initialization) Given an initial point $x_{0} \in R^{n}$ and an initial trust-region radius $\Delta_{0}>0.0<\eta_{1} \leq \eta_{2}<1$ and $0<\gamma_{1} \leq \gamma_{2}<1$. Set $k:=0$.

Step 1. (Model definition) Define a model function $m_{k}$ in $\mathfrak{I}_{k}$, where $\mathfrak{I}_{k}=\left\{x \in R^{n} \mid\left\|x-x_{k}\right\| \leq \Delta_{k}\right\}$.

Step 2. (Step calculation) Compute a trial step $s_{k}$ for solving trust region subproblem (4).

Step 3. (Updating iterate point) Compute $f\left(x_{k}+s_{k}\right)$ and the ratio

$$
\rho_{k}=\frac{f\left(x_{k}\right)-f\left(x_{k}+s_{k}\right)}{m_{k}\left(x_{k}\right)-m_{k}\left(x_{k}+s_{k}\right)} .
$$

then,

$$
x_{k+1}= \begin{cases}x_{k}+s_{k}, & \text { if } \rho_{k} \geq \eta_{1}, \\ x_{k}, & \text { if } \rho_{k}<\eta_{1} .\end{cases}
$$

Step 4. (Updating trust-region radius) Set

$$
\Delta_{k+1} \in \begin{cases}{\left[\Delta_{k}, \infty\right),} & \text { if } \rho_{k}>\eta_{2}, \\ {\left[\gamma_{2} \Delta_{k}, \Delta_{k}\right),} & \text { if } \rho_{k} \in\left[\eta_{1}, \eta_{2}\right], \\ {\left[\gamma_{1} \Delta_{k}, \gamma_{2} \Delta_{k}\right),} & \text { if } \rho_{k}<\eta_{1} .\end{cases}
$$

Set $k:=k+1$, and go to Step 1 .

In the algorithm BTR, we do not give a formal stopping criterion. In practice, the stopping criterion can be installed in Step 1, such as

$$
\left\|g_{k}\right\| \leq \text { eps or } k>k_{\max },
$$

where eps denotes the precision, and $k_{\max }$ denotes the maximal number of iterations.

If $x^{*}$ is a local minimizer of the problem (1), then $g\left(x^{*}\right)=0$. Motivated by the filter method, we set $\|g(x)\|$ to be the measure of the iterate. Now we intro- duce some definitions about the multi-dimensional filter.

Definition 2.1 We say that a point $x_{k}$ dominates another point $x_{l}$, if

$$
\left|g_{k j}\right| \leq\left|g_{l j}\right| \text { for all } j=1,2, \cdots n \text {. }
$$

Definition 2.2 A multi-dimensional filter $F$ is a list of n-tuples of the form $\left(g_{k 1}, \cdots, g_{k n}\right)$ and if $g_{k}, g_{l} \in F$, then there exist two indices $j_{1}, j_{2} \in\{1,2, \cdots n\}, j_{1} \neq j_{2}$ such that

$$
\left|g_{l j_{1}}\right|<\left|g_{k j_{1}}\right| \text { and }\left|g_{k j_{2}}\right|<\left|g_{l j_{2}}\right| \text {. }
$$

Definition 2.3 The iterate point $x_{k}$ is acceptable for the filter $F$ if and only if for all $g_{l} \in F$, there exists $j \in\{1,2, \cdots n\}$ such that

$$
\left|g_{k j}\right| \leq\left|g_{l j}\right|-\gamma_{g}\left\|g_{l}\right\|, \gamma_{g} \in(0,1 / \sqrt{n}) .
$$

Definition 2.4 If the iterate point $x_{k}$ is acceptable, then it is added to the filter and any iterates in the filter that are dominated by the new iterate are removed, which is called the modification of the filter.

Combining with the filter technique and the retrospective idea, we describe our algorithm as follows.

\subsection{Algorithm RFTR (Retrospective Filter Trust-Region Algorithm)}

Step 0. (Initialization) An initial point $x_{0} \in R^{n}$ and an initial trust-region radius $\Delta_{0}>0$ are given.

$$
\begin{aligned}
& \gamma_{g} \in(0,1 / \sqrt{n}), 0<\eta_{1}<1,0<\tilde{\eta}_{1} \leq \tilde{\eta}_{2}<1, \\
& 0<\gamma_{1} \leq \gamma_{2}<1 \leq \gamma_{3} .
\end{aligned}
$$

Set the initial filter $F$ to be the empty set and set $k:=0$.

Step 1. (Model definition) Define a model function $m_{k}$ in $\mathfrak{I}_{k}$, where $\mathfrak{I}_{k}=\left\{x \in R^{n} \mid\left\|x-x_{k}\right\| \leq \Delta_{k}\right\}$.

Step 2. (Updating retrospective trust-region radius) If $k=0$, then go to Step 3. If $x_{k}=x_{k-1}$, then choose $\Delta_{k} \in\left[\gamma_{1} \Delta_{k-1}, \gamma_{2} \Delta_{k-1}\right)$. Otherwise, compute the retrospective ratio

$$
\tilde{\rho}_{k}=\frac{f\left(x_{k-1}\right)-f\left(x_{k}\right)}{m_{k}\left(x_{k-1}\right)-m_{k}\left(x_{k}\right)} .
$$

Choose

$$
\Delta_{k} \in \begin{cases}{\left[\gamma_{1} \Delta_{k-1}, \gamma_{2} \Delta_{k-1}\right),} & \text { if } \tilde{\rho}_{k}<\tilde{\eta}_{1}, \\ {\left[\gamma_{2} \Delta_{k-1}, \Delta_{k-1}\right),} & \text { if } \tilde{\rho}_{k} \in\left[\tilde{\eta}_{1}, \tilde{\eta}_{2}\right], \\ {\left[\Delta_{k-1}, \gamma_{3} \Delta_{k-1}\right),} & \text { if } \tilde{\rho}_{k}>\tilde{\eta}_{2} .\end{cases}
$$

Step 3. (Step calculation) Compute a trial step $s_{k}$ for solving trust region subproblem (4) and $x_{k}^{+}=x_{k}+s_{k}$. 
Step 4. (Updating iterate point) Compute

$$
\rho_{k}=\frac{f\left(x_{k}\right)-f\left(x_{k}^{+}\right)}{m_{k}\left(x_{k}\right)-m_{k}\left(x_{k}^{+}\right)} .
$$

Case 1: If $\rho_{k} \geq \eta_{1}$, then $x_{k+1}=x_{k}^{+}$.

Case 2: If $\rho_{k}<\eta_{1}$ and $x_{k}^{+}$is accepted by the filter $F$, then $x_{k+1}=x_{k}^{+}$and add $g_{k}^{+}=g\left(x_{k}^{+}\right)$into the filter $F$.

Case 3: If $\rho_{k}<\eta_{1}$ and $x_{k}^{+}$is not accepted by the filter $F$, then $x_{k+1}=x_{k}$.

Step 5. Set $k:=k+1$, go to Step 1 .

Similar to the algorithm BTR, the stopping criterion can be installed in Step 1, such as

$$
\left\|g_{k}\right\| \leq e p s \text { or } k>k_{\max },
$$

where eps denotes the precision, and $k_{\max }$ denotes the maximal number of iterations. The Hessian matrix in the model function $m_{k}$ can be obtained by BFGS updating formula or set $\nabla_{x x} m_{k}\left(x_{k}+s\right)=\nabla_{x x} f\left(x_{k}\right)$ for all $s$ such that $x_{k}+s \in \mathfrak{I}_{k}$.

In the algorithm RFTR, the retrospective idea and the filter technique are two important characteristics. The retrospective ratio uses the information at the current iterate and the last iterate to adjust the trust-region radius, which can give the more effective estimation of trust region radius. The filter technique relaxes the condition of accepting a trial step comparing with the usual trust region method, which improves the effectiveness of the algorithm in some sense. From the algorithm RFTR, if the trial point is not accepted (Case 3 in Step 5 occurs), then the algorithm is similar to the basic trust-region algorithm, whose difference is just that we use the retrospective idea in the algorithm RFTR. However, if the trial point is accepted by the algorithm (Case 1 or Case 2 in Step 5 occurs), the retrospective idea and the filter technique all play the roles.

At the iterate $x_{k}$, if $x_{k+1}=x_{k}+s_{k} \neq x_{k}$, then the iterate is called the successful iterate and the iteration index $x_{k}$ is called successful iteration.

\section{Basic Assumptions and Lemmas}

In this section, we present the global convergence analysis of the algorithm RFTR. We make the following assumptions.

A1 The all iterates $x_{k}$ remain in a closed and bounded convex set $\Omega$.

A2 $f: R^{n} \rightarrow R$ is a twice continuously differentiable function.

A3 The model function $m_{k}$ is first-order coherent with the function $f$ at the iterate $x_{k}$, i.e., their values and gradients are equal at $x_{k}$ for all $k$,

$$
m_{k}\left(x_{k}\right)=f\left(x_{k}\right), \nabla_{x} m_{k}\left(x_{k}\right)=\nabla_{x} f\left(x_{k}\right) .
$$

A4 The Hessian matrix of the model function $\nabla_{x x} m_{k}$ is uniformly bounded, i.e., there exists a constant $k_{\text {umh }} \geq 1$ such that

$$
\left\|\nabla_{x x} m_{k}(x)\right\| \leq k_{u m h}-1,
$$

holds for all $x \in R^{n}$ and all $k$.

Generally speaking, we do not need the global solution of the trust region subproblem. We only expect to decrease the model at least as much as at the Cauchy point. Therefore, we make the following assumption on the solution $s_{k}$ of the trust region subproblem (4).

A5 There exists a constant $k_{m d c}$, for all $k$,

$$
\begin{aligned}
& m_{k}\left(x_{k}\right)-m_{k}\left(x_{k}+s_{k}\right) \\
& \geq k_{\text {mdc }} \max \left\{\left\|g_{k}\right\| \min \left\{\frac{\left\|g_{k}\right\|}{\beta_{k}}, \Delta_{k}\right\},\left|\tau_{k}\right| \min \left\{\tau_{k}^{2}, \Delta_{k}^{2}\right\}\right\},
\end{aligned}
$$

where

$$
\begin{aligned}
& \beta_{k}=1+\max _{x \in \mathfrak{I}_{k}}\left\|\nabla_{x x} m_{k}\right\|, \\
& \mathfrak{I}_{k}=\left\{x \in R^{n} \mid\left\|x-x_{k}\right\| \leq \Delta_{k}\right\}, \\
& \tau_{k}=\min \left\{0, \lambda_{\text {min }}\left(\nabla_{x x} m_{k}\left(x_{k}\right)\right)\right\} .
\end{aligned}
$$

By the assumptions $\mathrm{A} 1$ and $\mathrm{A} 2$, the Hessian matrix $\nabla_{x x} f(x)$ is uniformly bounded on $\Omega$, i.e., there exists a positive constant $k_{u f h}$ such that, for all $x \in \Omega$,

$$
\left\|\nabla_{x x} f(x)\right\| \leq k_{u f h} .
$$

Now we study the global convergence of the algorithm RFTR. First we give a bound on the difference between the objective function and the model function $m_{k}$ at the iterate $x_{k-1}$ and $x_{k}$. The proof of the following result is similar to Theorem 3.1 in [25].

Lemma 3.1 [25] Suppose A1-A4 hold, then exists a positive constant $k_{u b h}$,

$$
\left|f\left(x_{k}\right)-m_{k-1}\left(x_{k-1}\right)\right| \leq k_{u b h} \Delta_{k-1}^{2}
$$

and if iteration $k-1$ is successful, that

$$
\left|f\left(x_{k-1}\right)-m_{k}\left(x_{k-1}\right)\right| \leq k_{u b h} \Delta_{k-1}^{2}
$$

where $k_{u b h}=\max \left\{k_{u f h}, k_{u m h}\right\}$.

As the retrospective ratio $\tilde{\rho}_{k}$ uses the reduction in $m_{k}$ instead of the reduction in $m_{k-1}$, we need to compare their difference, which is provided by the next Lemma.

Lemma 3.2 [25] Suppose A1-A4 hold, then for every successful iteration $k-1$,

$$
\begin{aligned}
& \mid\left(m_{k-1}\left(x_{k-1}\right)-m_{k-1}\left(x_{k}\right)\right) \\
& -\left(m_{k}\left(x_{k-1}\right)-m_{k}\left(x_{k}\right)\right) \mid \leq 2 k_{u b h} \Delta_{k-1}^{2}
\end{aligned}
$$


We conclude from this result that the denominators in the expression of $\tilde{\rho}_{k}$ and $\rho_{k}$ are the same order as the error between the objective function and the model function. Similar to Theorem 6.4.2 in [13], we obtain the next result.

Lemma 3.3 Suppose A1-A5 hold, $g_{k-1} \neq 0$ and $\Delta_{k-1}$ satisfies that

$$
\Delta_{k-1} \leq \min \left\{1-\eta_{1}, \frac{1-\tilde{\eta}_{2}}{3-2 \tilde{\eta}_{2}}\right\} \frac{k_{m d c}}{k_{u b h}}\left\|g_{k-1}\right\|
$$

Then iteration $k-1$ is successful and

$$
\Delta_{k} \geq \Delta_{k-1} .
$$

Proof. It follows from $k_{m d c}, \eta_{1} \in(0,1)$ and the assumptions $\mathrm{A} 3$ and $\mathrm{A} 5$ that $\beta_{k-1} \leq k_{u b h}$. By (7),

$$
\Delta_{k-1}<\frac{\left\|g_{k-1}\right\|}{\beta_{k-1}} .
$$

By the assumption A5, we have that

$$
\begin{aligned}
m_{k-1}\left(x_{k-1}\right)-m_{k-1}\left(x_{k}\right) & \geq k_{\text {mdc }}\left\|g_{k-1}\right\| \min \left\{\frac{\left\|g_{k-1}\right\|}{\beta_{k-1}}, \Delta_{k-1}\right\} \\
& \geq k_{m d c}\left\|g_{k-1}\right\| \Delta_{k-1}
\end{aligned}
$$

On the other hand, it follows from (5) and (7) that

$$
\begin{aligned}
& \left|\rho_{k-1}-1\right|=\left|\frac{f\left(x_{k}\right)-m_{k-1}\left(x_{k}\right)}{m_{k-1}\left(x_{k-1}\right)-m_{k-1}\left(x_{k}\right) \mid}\right| \\
& \leq \frac{k_{u b h}}{k_{m d c}\left\|g_{k-1}\right\|} \Delta_{k-1} \leq 1-\eta_{1} .
\end{aligned}
$$

Thus, $\rho_{k-1} \geq \eta_{1}$, i.e., the iteration $k-1$ is successful. Next we proof the second part of the conclusion.

By $k_{m d c}, \tilde{\eta}_{2} \in(0,1)$, we have

$$
\frac{1-\tilde{\eta}_{2}}{3-2 \tilde{\eta}_{2}}<\frac{1}{2} \text { and } k_{m d c} \frac{1-\tilde{\eta}_{2}}{3-2 \tilde{\eta}_{2}}<1
$$

The conditions (7), (9) and the definition of $\beta_{k-1}$ in the assumption A5 imply that

$$
\Delta_{k-1}<\frac{1}{2} \frac{k_{m d c}}{k_{u b h}}\left\|g_{k-1}\right\| \text { and } \Delta_{k-1}<\frac{\left\|g_{k-1}\right\|}{\beta_{k-1}} .
$$

Combining (8) and Lemma 3.2, we can conclude that

$$
\begin{aligned}
\left|m_{k}\left(x_{k-1}\right)-m_{k}\left(x_{k}\right)\right| & \geq\left|m_{k-1}\left(x_{k-1}\right)-m_{k-1}\left(x_{k}\right)\right|-2 k_{u b h} \Delta_{k-1}^{2} \\
& \geq k_{m d c} \| g_{k-1}|| \Delta_{k-1}-2 k_{u b h} \Delta_{k-1}^{2}
\end{aligned}
$$

By (6) and (10),

$$
\left|\tilde{\rho}_{k}-1\right|=\left|\frac{f\left(x_{k-1}\right)-m_{k}\left(x_{k-1}\right)}{m_{k}\left(x_{k-1}\right)-m_{k}\left(x_{k}\right)}\right| \leq \frac{k_{u b h} \Delta_{k-1}}{k_{m d c}|| g_{k-1} \mid-2 k_{u b h} \Delta_{k-1}} .
$$

(7) implies that

$$
\left(3-2 \tilde{\eta}_{2}\right) k_{u b h} \Delta_{k-1} \leq\left(1-\tilde{\eta}_{2}\right) k_{m d c}\left\|g_{k-1}\right\| .
$$

i.e.,

$$
k_{u b h} \Delta_{k-1} \leq\left(1-\tilde{\eta}_{2}\right)\left(k_{m d c}\left\|g_{k-1}\right\|-2 k_{u b h} \Delta_{k-1}\right) .
$$

Therefore, $\left|\tilde{\rho}_{k}-1\right| \leq\left(1-\tilde{\eta}_{2}\right)$, i.e., $\tilde{\rho}_{k} \geq \tilde{\eta}_{2}$.

As a consequence of this property, we may now prove that the trust region radius cannot become too small as long as a first-order critical point is not approached. The technique of the proof is similar to Theorem 3.4 in [25] and Theorem 6.4.3 in [13].

Lemma 3.4 [13,25] Suppose A1-A5 hold. Suppose, furthermore, that there exists a constant $k_{l b g}>0$ such that $\left\|g_{k}\right\| \geq k_{l b g}$ for all $k$. Then there is a constant $k_{l b d}$ such that $\Delta_{k} \geq k_{l b d}$ for all $k$.

\section{First Order Convergence}

Assume that $\left\{x_{k}\right\}$ is an infinite sequence generated by Algorithm RFTR. Under the assumptions (A1)-(A5), we discuss the first order convergence of the sequence $\left\{x_{k}\right\}$. At first, we define the following sets.

The set of successful iteration index

$$
S=\left\{k \mid x_{k+1}=x_{k}+s_{k}\right\} .
$$

The set of the iteration index which is added to the filter

$$
\begin{aligned}
A= & \left\{k \mid g_{k}^{+} \text {or the iterate } x_{k}^{+}=x_{k}+s_{k}\right. \\
& \text { is added to the filter } F\} .
\end{aligned}
$$

The set of the iteration index which satisfies sufficient descent condition

$$
D=\left\{k \mid \rho_{k} \geq \eta_{1}\right\} .
$$

$|S|$ denotes the cardinal number of the set $S$. We now establish the criticality of the limit point of the sequence of the iterates when there are only finitely many successful iterations.

Theorem 4.1 Suppose A1-A5 hold and $|S|<+\infty$, then there exists an index $k_{0}$ such that $x_{k_{0}} \equiv x^{*}$ and $x^{*}$ is a first-order critical point.

Proof. Let $k_{0}$ be the last successful iteration index, then

$$
x_{k_{0}}=x_{k_{0}+j} \text { and } \rho_{k_{0}+j}<\eta_{1}, \forall j=1,2, \cdots .
$$

From Step 2 of the algorithm RFTR, we have that 


$$
\Delta_{k_{0}+j} \leq \gamma_{2} \Delta_{k_{0}+j-1} \leq \cdots \leq \gamma_{2}^{j} \Delta_{k_{0}}
$$

Thus,

$$
\lim _{j \rightarrow \infty} \Delta_{k_{0}+j}=0 .
$$

It follows from Lemma 3.4 that $\left\|g_{k_{0}}\right\|=0$.

Next, we consider the case that there are infinitely many successful iteration. From the algorithm RFTR, we know that $A \subseteq S$. Therefore we consider the following two cases.

1) There are infinitely many filter iterations, i.e., $|A|=+\infty$.

2) There are finitely many filter iterations, i.e., $|A|<$ $+\infty$.

First, we have the following result.

Theorem 4.2 Suppose A1-A5 hold and $|A|=|S|=+\infty$, then

$$
\liminf _{k \rightarrow \infty}\left\|g_{k}\right\|=0
$$

Proof. Suppose, by contradiction, that the result is not true, then there exists a positive constant $k_{l b g}$ such that

$$
\left\|g_{k}\right\| \geq k_{l b g}>0
$$

holds for all $k$. Denote the index set $A=\left\{k_{i}\right\}$. It follows from the assumption A1-A5 that $\left\{\left\|g_{k}\right\|\right\}$ is bounded. So there exists a subsequence $\left\{k_{i_{l}}+1\right\}$ of $\left\{k_{i}+1\right\}$ which satisfies

$$
\lim _{l \rightarrow \infty}\left\|g_{k_{i l}+1}\right\|=\left\|g_{\infty}\right\| \geq k_{l b g}
$$

By the definition of $k_{i_{l}}$, the iterate point $x_{k_{i j}+1}$ is accepted by the filter $F$, and for every $l>1$ there exists $j_{l} \in\{1,2, \cdots, n\}$ such that

$$
\left|g_{k_{i l}+1, j_{l}}\right|-\left|g_{k_{i-1}+1, j_{l}}\right| \leq-\gamma_{g} \| g_{k_{i l-1}+1}|| .
$$

Since there is only finite choices of $j_{l}$, without loss of generality, we set $j_{l}=j$. In (12), we follows from $l \rightarrow+\infty$ and (11) that

$$
0 \leftarrow\left|g_{k_{i l}+1, j}\right|-\left|g_{k_{i l-1}+1, j}\right| \leq-\gamma_{g} k_{l b g}<0,
$$

which is a contradiction. Thus the result is proved.

Now, we give the result when $|A|<+\infty$.

Theorem 4.3 Suppose A1-A5 hold and $|S|=+\infty$, $|A|<+\infty$, then

$$
\liminf _{k \rightarrow \infty}\left\|g_{k}\right\|=0 \text {. }
$$

Proof. Suppose, by contradiction, that the result is not true, then there exists a positive constant $k_{l b g}$ such that (11) holds. Since $|A|<+\infty$, by the algorithm RFTR, we have that $\rho_{k} \geq \eta_{1}$ holds for all sufficiently large index $k \in S$. Denote

$$
\sigma_{k}=|\{p, p+1, \cdots, k\} \cap S| .
$$

It follows from the assumption A5, Lemma 3.4 and $\rho_{k} \geq \eta_{1}$ that

$$
\begin{aligned}
& f\left(x_{p}\right)-f\left(x_{k+1}\right)=\sum_{\substack{j=p \\
j \in S}}^{k}\left(f\left(x_{j}\right)-f\left(x_{j+1}\right)\right) \\
& \geq \sigma_{k} \eta_{1} k_{m d c} k_{l b g} \min \left\{\frac{k_{l b g}}{k_{u m h}}, k_{l b d}\right\} .
\end{aligned}
$$

as long as $p, k$ is sufficiently large. $|S|=+\infty$ and $|A|<+\infty$ imply that $\sigma_{k}$ may be large arbitrarily, which contradicts with the fact that $\left\{f\left(x_{k}\right)\right\}$ is bounded.

By Theorem 4.1, Theorem 4.2 and Theorem 4.3, there exists at least one limit point of the sequence $\left\{x_{k}\right\}$ of iterates generated by the algorithm RFTR which is a first-order critical point.

\section{Second Order Convergence}

We now exploit second-order information on the objective function to discuss the second order convergence of the sequence. We therefore introduce the following additional assumptions.

A6 The model is asymptotically second-order coherent with the objective function near first-order critical points, i.e.,

$\lim _{k \rightarrow \infty}\left\|\nabla_{x x} f\left(x_{k}\right)-\nabla_{x x} m_{k}\left(x_{k}\right)\right\|=0$ where $\lim _{k \rightarrow \infty}\left\|g_{k}\right\|=0$.

A7 There exists a constant $k_{\text {lch }}$ such that, for all $k$,

$$
\left\|\nabla_{x x} m_{k}(x)-\nabla_{x x} m_{k}(y)\right\| \leq k_{l c h}\|x-y\| .
$$

for all $x, y \in \mathfrak{J}_{k}$.

Lemma 5.1 Suppose that A1-A7 hold. Suppose also that there exists a sequence $\left\{k_{i}\right\}$ and a constant $k_{m q d}>0$ such that

$$
m_{k_{i}}\left(x_{k_{i}}\right)-m_{k_{i}}\left(x_{k_{i}}+s_{k_{i}}\right) \geq k_{m q d}\left\|s_{k_{i}}\right\|^{2}>0
$$

for all $i$ sufficiently large. Finally, suppose that $\lim _{i \rightarrow \infty}$ $\left\|s_{k_{i}}\right\|=0$, then iteration $k_{i}$ is successful and

$$
\tilde{\rho}_{k_{i}+1} \geq \tilde{\eta}_{2} \text { and } \Delta_{k_{i}+1} \geq \Delta_{k_{i}}
$$

for all $i$ sufficiently large.

Proof. We first deduce that every iterations $k_{i}$ is successful for $i$ sufficiently large. By the mean value theorem and (13), for some $\xi_{k}$ and $\varsigma_{k}$ in the segment $\left[x_{k_{i}}, x_{k_{i}+1}\right]$, 


$$
\begin{aligned}
\left|\rho_{k_{i}}-1\right|= & \left|\frac{f\left(x_{k_{i}+1}\right)-m_{k_{i}}\left(x_{k_{i}+1}\right)}{m_{k_{i}}\left(x_{k_{i}}\right)-m_{k_{i}}\left(x_{k_{i}+1}\right)}\right| \\
\leq & \frac{1}{2 k_{m q d} \mid s_{k_{i}}}||^{2}\left|s_{k_{i}}^{T}\left(\nabla_{x x} f\left(\xi_{k_{i}}\right)-\nabla_{x x} m_{k_{i}}\left(s_{k_{i}}\right)\right) s_{k_{i}}\right| \\
\leq & \frac{1}{2 k_{m q d}}\left(\| \nabla_{x x} f\left(\xi_{k_{i}}\right)-\nabla_{x x} f\left(x_{k_{i}}\right)||\right. \\
& +\left\|\nabla_{x x} f\left(x_{k_{i}}\right)-\nabla_{x x} m_{k_{i}}\left(x_{k_{i}}\right)\right\| \\
& \left.+\left\|\nabla_{x x} m_{k_{i}}\left(x_{k_{i}}\right)-\nabla_{x x} m_{k_{i}}\left(s_{k_{i}}\right)\right\|\right),
\end{aligned}
$$

When $i$ goes to infinity, by our assumption that $\left\|s_{k_{i}}\right\|$ tends to 0 , and the bounds

$$
\left\|\xi_{k_{i}}-x_{k_{i}}\right\| \leq\left\|s_{k_{i}}\right\| \text { and }\left\|\varsigma_{k_{i}}-x_{k_{i}}\right\| \leq\left\|s_{k_{i}}\right\| .
$$

Combining the assumption $\mathrm{A} 2$ and $\mathrm{A} 7$, the first and third terms of the last right-hand side tend to 0 . Meanwhile, the second tends to 0 because of the assumption A6 and Theorem 4.1, 4.2, 4.3. As a consequence, $\rho_{k_{i}}$ tends to 1 . when $i$ goes to infinity, and thus larger than $\eta_{1}$ for $i$ sufficiently large.

The residual proof is similar to Lemma 3.8 in [25].

Theorem 5.2 Suppose that A1-A7 hold and that the complete sequence of iterates $\left\{x_{k}\right\}$ converges to the unique limit point $x^{*}$. Then $x^{*}$ is a second order critical point of (1).

Proof. By Theorem 4.1, 4.2, 4.3, $g\left(x^{*}\right)=0$. We suppose, by contradiction, that $\tau_{*}=\lambda_{\min }\left(\nabla_{x x} f\left(x^{*}\right)\right)<0$, by the assumption $\mathrm{A} 6$, there exists $k_{0}$ such that, for all $k \geq k_{0}, \tau_{k}=\lambda_{\min }\left(\nabla_{x x} m_{k}\left(x_{k}\right)\right)<\frac{1}{2} \tau_{*}$. It follows from the assumption A5 that

$$
m_{k}\left(x_{k}\right)-m_{k}\left(x_{k}+s_{k}\right) \geq \frac{1}{2} k_{m d c}\left|\tau_{*}\right| \min \left\{\frac{1}{4}\left|\tau_{*}^{2}\right|, \Delta_{k}^{2}\right\}
$$

hold for all $k \geq k_{0}$. Meanwhile, by the assumption we know that $s_{k}=x_{k+1}-x_{k}$ tends to 0 . Thus, there exists $k_{1} \geq k_{0}$ such that, for all $k \geq k_{1},\left\|s_{k}\right\| \leq \min \left\{1 / 2\left|\tau_{*}\right|, \Delta_{k}\right\}$. By Lemma 5.1, there exists $k_{2} \geq k_{1}$ such that, for all $k \geq k_{2}$, we deduce that $\rho_{k} \geq \eta_{1}, \tilde{\rho}_{k+1} \geq \tilde{\eta}_{2}$ and $\Delta_{k+1} \geq \Delta_{k}$. Thus,

$$
\begin{aligned}
f\left(x_{k}\right)-f\left(x_{k+1}\right) & \geq \eta_{1}\left(m_{k}\left(x_{k}\right)-m_{k}\left(x_{k}+s_{k}\right)\right) \\
& \geq \frac{1}{2} \eta_{1} k_{m d c}\left|\tau_{*}\right| \min \left\{\frac{1}{4}\left|\tau_{*}^{2}\right|, \Delta_{k}^{2}\right\},
\end{aligned}
$$

which follows from $\lim _{k \rightarrow \infty} x_{k}=x^{*}$ that $\lim _{k \rightarrow \infty} \Delta_{k}=0$. This contradicts with $\Delta_{k+1} \stackrel{k \rightarrow \infty}{\stackrel{\infty}{\Delta}} \Delta_{k}$ for all $k \geq k_{2}^{*}$. Thus, $\tau_{*} \geq 0$ and therefore $x^{*}$ is a second-order critical point of (1).

\section{Numerical Experiments}

In this section, a preliminary numerical test of the algorithm BTR, the algorithm FTR [22], the algorithm RTR [25] and the algorithm FTR are given. The Matlab codes (Version 7.4.0.287 R2007a) were written corresponding to those algorithms. For the numerical tests, we use the following trust-region radius updated form which is proposed in Conn et al. [13].

$$
\Delta_{k+1}= \begin{cases}\gamma_{1}\left\|s_{k}\right\|, & \text { if } \tilde{\rho}_{k+1}<\tilde{\eta}_{1}, \\ \Delta_{k}, & \text { if } \tilde{\rho}_{k+1} \in\left[\tilde{\eta}_{1}, \tilde{\eta}_{2}\right], \\ \max \left(\gamma_{3}\left\|s_{k}\right\|, \Delta_{k}\right), & \text { if } \tilde{\rho}_{k+1}>\tilde{\eta}_{2} .\end{cases}
$$

and the following parameter settings $[21,28]$.

$$
\begin{gathered}
\Delta \gamma_{1}=0.25, \gamma_{3}=3.5, \tilde{\eta}_{1}=\eta_{1}=0.0001, \tilde{\eta}_{2}=\eta_{2}=0.99, \\
\Delta_{0}=1, \gamma_{g}=\min \{0.001,1 /(2 \sqrt{n})\} .
\end{gathered}
$$

The Hessian matrix of the model function is $\nabla_{x x} m_{k}(x)=\nabla_{x x} f\left(x_{k}\right)$. The termination criterion is as following,

$$
\left\{\left\|g_{k}\right\| \leq 10^{-6} \sqrt{n} \text { or } k>k_{\max }\right\}, k_{\max }=1000,
$$

where $k_{\max }$ denotes the maximal iteration number.

We choose 24 test problems from [29], where "S201" means problem 201 in Schittkowski (1987) collection [29], 12 test problems from CUTE $[25,30]$ and the famous Extended Rosenbrock test problem. In the following tables, "n" means the test problem's dimension, "nBTR, nFTR, nRTR, nRFTR" mean the number of iterations of the algorithm BTR, the algorithm FTR, the algorithm RTR and the algorithm RFTR, respectively. "ng1, ng2, ng3, ng4" mean the number of gradient evaluations of the algorithm BTR, the algorithm FTR, the algorithm RTR and the algorithm RFTR, respectively.

" $r$ " means the rank of the number of iterations of the algorithm RFTR among the four algorithms, whose values is in $\{1,2,3,4\}$, where " 1 " means that the number of iterations of the algorithm RFTR is the smallest among the four algorithms, so the algorithm RFTR is the best one among the four algorithms. " 4 " means that the number of iterations of the algorithm RFTR is the largest among the four algorithms, so the algorithm RFTR is the worst one among the four algorithms. "F" means that the algorithm does not stop when the maximal iteration number is achieved.

In Table 1, there are 20 test problems whose iteration number is the smallest, 2 test problems whose rank is second, 2 test problems whose iteration number is the largest among 24 test problems. The numerical results 
Table 1. Reports the numerical results on 24 test problems from [29].

\begin{tabular}{|c|c|c|c|c|c|c|c|c|c|c|}
\hline Problem & $\mathrm{n}$ & nBTR & nFTR & nRTR & nRFTR & ng1 & ng2 & ng3 & ng4 & $\mathrm{r}$ \\
\hline S201 & 2 & 34 & 34 & 34 & 34 & 35 & 35 & 35 & 35 & 1 \\
\hline S202 & 2 & 6 & 6 & 6 & 6 & 7 & 7 & 7 & 7 & 1 \\
\hline S203 & 2 & 6 & 6 & 6 & 6 & 7 & 7 & 7 & 7 & 1 \\
\hline S205 & 2 & 8 & 8 & 8 & 8 & 9 & 9 & 9 & 9 & 1 \\
\hline S206 & 2 & 4 & 4 & 4 & 4 & 5 & 5 & 5 & 5 & 1 \\
\hline S207 & 2 & 9 & 7 & 11 & 7 & 8 & 9 & 10 & 9 & 1 \\
\hline S208 & 2 & 39 & 17 & 27 & 17 & 27 & 23 & 26 & 23 & 1 \\
\hline S209 & 2 & 108 & 45 & 99 & 53 & 86 & 55 & 93 & 61 & 2 \\
\hline S210 & 2 & 424 & 169 & 460 & 176 & 365 & 195 & 450 & 185 & 2 \\
\hline S211 & 2 & 37 & 13 & 37 & 12 & 29 & 17 & 32 & 16 & 1 \\
\hline S212 & 2 & 13 & 14 & 12 & 14 & 11 & 16 & 11 & 16 & 4 \\
\hline S213 & 2 & 27 & 27 & 27 & 27 & 28 & 28 & 28 & 28 & 1 \\
\hline S240 & 3 & 5 & 5 & 5 & 5 & 6 & 6 & 6 & 6 & 1 \\
\hline S241 & 3 & 13 & 13 & 13 & 13 & 14 & 14 & 14 & 14 & 1 \\
\hline S246 & 3 & 10 & 7 & 10 & 7 & 10 & 9 & 10 & 9 & 1 \\
\hline S256 & 4 & 15 & 15 & 15 & 15 & 16 & 16 & 16 & 16 & 1 \\
\hline S260 & 4 & 69 & 33 & 53 & 33 & 47 & 37 & 48 & 37 & 1 \\
\hline S261 & 4 & 13 & 15 & 13 & 15 & 13 & 17 & 13 & 17 & 4 \\
\hline S271 & 6 & 2 & 2 & 2 & 2 & 3 & 3 & 3 & 3 & 1 \\
\hline S273 & 6 & 10 & 10 & 10 & 10 & 11 & 11 & 11 & 11 & 1 \\
\hline S274 & 2 & 2 & 2 & 2 & 2 & 3 & 3 & 3 & 3 & 1 \\
\hline S275 & 4 & 2 & 2 & 2 & 2 & 3 & 3 & 3 & 3 & 1 \\
\hline S276 & 6 & 2 & 2 & 2 & 2 & 3 & 3 & 3 & 3 & 1 \\
\hline S308 & 2 & 11 & 9 & 11 & 9 & 10 & 11 & 10 & 11 & 1 \\
\hline
\end{tabular}

Table 2. Reports the numerical results on the famous Extended Rosenbrock test problem.

\begin{tabular}{cccccccccc}
\hline $\mathrm{n}$ & $\mathrm{nBTR}$ & $\mathrm{nFTR}$ & $\mathrm{nRTR}$ & $\mathrm{nRFTR}$ & $\mathrm{ng} 1$ & $\mathrm{ng} 2$ & $\mathrm{ng} 3$ & $\mathrm{ng} 4$ & $\mathrm{r}$ \\
\hline 2 & 39 & 17 & 27 & 17 & 27 & 23 & 26 & 23 & 1 \\
10 & 36 & 22 & 31 & 22 & 28 & 29 & 29 & 29 & 1 \\
20 & 67 & 36 & 68 & 36 & 49 & 43 & 67 & 43 & 1 \\
30 & 83 & 41 & 60 & 42 & 62 & 56 & 58 & 55 & 2 \\
40 & 111 & 52 & 100 & 52 & 81 & 70 & 98 & 70 & 1 \\
50 & 150 & 69 & 130 & 69 & 405 & 93 & 127 & 91 & 1 \\
60 & 170 & 82 & 147 & 82 & 122 & 109 & 144 & 107 & 1 \\
70 & 199 & 102 & 162 & 101 & 141 & 140 & 159 & 135 & 1 \\
80 & 218 & 121 & 171 & 120 & 157 & 155 & 170 & 147 & 1 \\
90 & 248 & 128 & 209 & 121 & 177 & 171 & 207 & 150 & 1 \\
100 & 278 & 141 & 220 & 141 & 179 & 182 & 218 & 180 & 1 \\
150 & 397 & 207 & 296 & 213 & 284 & 276 & 293 & 274 & 2 \\
200 & 532 & 302 & 427 & 283 & 378 & 391 & 423 & 361 & 1 \\
250 & 647 & 374 & 507 & 373 & 464 & 478 & 504 & 474 & 1 \\
300 & 769 & 419 & 722 & 419 & 551 & 542 & 719 & 537 & 1 \\
350 & 900 & 507 & F & 501 & 644 & 653 & 996 & 627 & 1 \\
400 & F & 585 & F & 500 & 715 & 748 & 997 & 536 & 798 \\
450 & F & 640 & 878 & 630 & 712 & 834 & 873 & 994 & 1 \\
500 & F & 719 & F & 710 & 712 & 938 & 994 & 1 \\
\hline
\end{tabular}


Table 3. Reports the numerical results on 12 test problems from CUTE $[25,30]$.

\begin{tabular}{lccccccccccc}
\hline \multicolumn{1}{c}{ Problem } & $\mathrm{n}$ & $\mathrm{nBTR}$ & $\mathrm{nFTR}$ & $\mathrm{nRTR}$ & $\mathrm{nRFTR}$ & $\mathrm{ng} 1$ & $\mathrm{ng} 2$ & $\mathrm{ng} 3$ & $\mathrm{ng} 4$ & $\mathrm{r}$ \\
\hline ARHEAD & 100 & 5 & 5 & 5 & 5 & 6 & 6 & 6 & 6 & 1 \\
ROSNSCHN & 50 & 66 & 39 & 100 & 39 & 51 & 46 & 98 & 46 & 1 \\
COSINE & 100 & $\mathrm{~F}$ & $\mathrm{~F}$ & 34 & 36 & 986 & 1008 & 32 & 40 & 2 \\
DQDRTIC & 100 & 4 & 4 & 4 & 4 & 5 & 5 & 5 & 5 & 1 \\
ERRINROS & 50 & 52 & 67 & 48 & 27 & 39 & 78 & 44 & 33 & 1 \\
FLERCHCR & 100 & 29 & 29 & 29 & 29 & 30 & 30 & 30 & 30 & 1 \\
LIARWHD & 300 & 13 & 13 & 13 & 13 & 14 & 14 & 14 & 14 & 1 \\
LOGHAIRY & 2 & 61 & 64 & 55 & 31 & 51 & 66 & 50 & 33 & 1 \\
NONDIA & 100 & 24 & 24 & 24 & 24 & 25 & 25 & 25 & 25 & 1 \\
PFIT4LS & 3 & 271 & 291 & 309 & 233 & 217 & 323 & 300 & 245 & 1 \\
POWELLS & 4 & 15 & 15 & 15 & 15 & 16 & 16 & 16 & 16 & 1 \\
WOOD & 4 & 69 & 33 & 53 & 33 & 47 & 37 & 48 & 37 & 1 \\
\hline
\end{tabular}

show that the number for the algorithm RFTR to solve trust region subproblem is the smallest in total.

In Table 2, There are only 2 cases whose rank is second, the others all are the best. Moreover, the algorithm RFTR is more and more effective as the increase of the problem's dimension.

In Table 3, There are only 1 case whose rank is second, the others all are the best. Moreover, The retrospective idea takes effects on the Problems COSINE, ERRINROS, LOGHAIRY clearly.

\section{Conclusions and Perspectives}

Trust region method is very reliable and robust and has very strong convergence properties. It is a class of very effective algorithms for solving unconstrained optimization now. The basic trust region algorithm is the monotone descent algorithm, i.e., the value of the object function in the iterate sequence $\left\{x_{k}\right\}$ strictly decreases monotonically. If the iterates follow the bottom of curved narrow valleys, then the monotone descent algorithm converges very slowly. The idea of non-monotone method $[23,24]$ abandons the restriction of the descent property of the value of the object function, which allows the sequence of iterates to follow the bottom of curved narrow valleys much more loosely, which hopefully results in longer and more efficient steps.

Trust region method combines with the filter technique, which, in some sense, relaxes the monotonicity condition which accepts the trial step. The filter technique improves the numerical effect for some problems.

The new algorithm RFTR presented in this paper combines with the filter technique and the retrospective idea, which the number of the algorithm RFTR to solve trust region subproblem is decreased in total. On the other hand, our algorithm also looks like a self-adaptive method based on the trust-region framework. Meanwhile, our algorithm is not like the other algorithms about self-adaptive method [26,27] which need to compute the gradient value and function value at the auxiliary point, but may measure the acceptance of the previous iterate and the current iterate for the new and old model function, respectively, which keep the robustness property of the trust-region method.

\section{References}

[1] M. J. D. Powell, "A New Algorithm for Unconstrained Optimization,” In: J. B. Rosen, O. L. Mangasarian and K. Ritter, Eds., Nonlinear Programming, Academic Press, New York, 1970.

[2] K. Levenberg, "A Method for The Solution of Certain Nonlinear Problems in Least Squares," The Quarterly of Applied Mathematics, Vol. 2, No. 2, 1944, pp. 164-168.

[3] D. W. Marquardt, "An Algorithm for Least Squares Estimation of Nonlinear Inequalities," SIAM Journal on Applied Mathematics, Vol. 11, No. 2, 1963, pp. 431-441.

[4] M. J. D. Powell, "Convergence Properties of a Class of Minimization Algorithms," In: O. L. Mangasarian, R. R. Meyer and S. M. Robinson, Eds., Nonlinear Programming, Academic Press, New York, 1975, pp. 1-27.

[5] M. J. D. Powell, "On the Global Convergence of TrustRegion Algorithms for Unconstrained Optimization," Mathematical Programming, Vol. 29, No. 3, 1984, pp. 297-303.

[6] G. A. Schultz, R. B. Schnabei and R. H. Byrd, "A Family of Trust-Region-Based Algorithms for Unconstrained Minimization with Strong Global Convergence," SIAM Journal on Numerical Analysis, Vol. 22, No. 1, 1985, pp. 47-67. 
[7] D. C. Sorensen, "Newton's Method with a Model Trust Region Modifications," SIAM Journal on Numerical Analysis, Vol. 19, No. 2, 1982, pp. 409-426.

[8] J. J. Moŕe, "Recent Developments in Algorithms and Software for Trust Region Methods," In A. R. Bachem, M. Grotshel and B. Korte, Eds., Mathematical Programming: The State of the Art, Springer-Verlag, Berlin, 1983, pp. 258-287.

[9] Y. X. Yuan, "On the Convergence of Trust Region Algorithm," Mathematica Numerica Sinica, Vol. 16, No. 3, 1996, pp. 333-346.

[10] M. Lalee, J. Nocedal and T. Plantenga, "On the Implentation of an Algorithm for Large-Scale Equality Constrained Optimization," SIAM Journal on Optimization, Vol. 8, No. 3, 1998, pp. 682-706.

[11] A. Friedlander, J. M. Martinez and S. A. Santos, "A New Trust Region Algorithm for Bound Constrained Minimization," Applied Mathematics and Optimization, Vol. 30, No. 3, 1994, pp. 235-266.

[12] A. R. Conn, N. I. M. Gould and P. L. Toint, "Convergence Properties of Minimization Algorithms for Convex Constraints Using a Structured Trust Region," SIAM Journal on Optimization, Vol. 6, No. 4, 1996, pp. 10591086.

[13] A. R. Conn, N. I. M. Gould and P. L. Toint, "Trust Region Methods," MPS-SIAM Series on Optimization, SIAM, Philadelphia, 2000.

[14] R. Fletcher and S. Leyffer, "Nonlinear Programming without a Penalty Function," Mathematical Programming, Vol. 91, No. 2, 2002, pp. 239-269.

[15] R. Fletcher, N. I. M. Gould, S. Leyffer, P. L. Toint and A. Wächter, "Global Convergence of a Trust-Region SQPFilter Algorithm for General Nonlinear Programming," SIAM Journal on Optimization, Vol. 13, No. 3, 2002, pp. 635-659.

[16] R. Fletcher, S. Leyffer and P. L. Toint, "On the Global Convergence of a Filter-SQP Algorithm," SIAM Journal on Optimization, Vol. 13, No. 1, 2002, pp. 44-59.

[17] M. Ulbrich, S. Ulbrich and L. N. Vicente, "A Globally Convergent Primal Dual Interior-Point Filter Method for Nonconvex Nonlinear Programming," Mathematical Programming, Vol. 100, No. 2, 2003, pp. 379-410.

[18] A. Wächter and L. T. Biegler, "Line Search Filter Methods for Nonlinear Programming: Motivation and Global Convergence," SIAM Journal on Optimization, Vol. 16, No. 1, 2005, pp. 1-31.
[19] A. Wächter and L. T. Biegler, "Line Search Filter Methods for Nonlinear Programming: Local Convergence," SIAM Journal on Optimization, Vol. 16, No. 1, 2005, pp. $32-48$.

[20] R. Fletcher, S. Leyffer and P. L. Toint, "A Brief History of Filter Methods," SIAG/OPT Views and News, Vol. 18, No. 1, 2006, pp. 2-12.

[21] N. I. M. Gould, C. Sainvitu and P. L. Toint, "A FilterTrust-Region Method for Unconstrained Optimization," SIAM Journal on Optimization, Vol. 16, No. 2, 2005, pp. 341-357.

[22] W. H. Miao and W. Y. Sun, "A Filter Trust-Region Method for Unconstrained Optimization," Numerical Mathematics - A Journal of Chinese Universities. Gaodeng Xuexiao Jisuan Shuxue Xuebao, Vol. 29, No. 1, 2007, pp. 88-96.

[23] L. Grippo, F. Lampariello and S. Lucidi, "A Nonmonotone Line Search Technique for Newton's Methods," SIAM Journal on Numerical Analysis, Vol. 23, No. 4, 1986, pp. 707-716.

[24] P. L. Toint, "Non-Monotone Trust-Region Algorithms for Nonlinear Optimization Subject to Convex Constraints," Mathematical Programming, Vol. 77, No. 1, 1997, pp. 69-94.

[25] F. Bastin, V. Malmedy, M. Mouffe, P. L. Toint and D. Tomanos, "A Retrospective Trust-Region Method for Unconstrained Optimization," Mathematical Programming, Vol. 123, No. 2, 2010, pp. 395-418.

[26] A. Sartenaer, "Automatic Determination of an Initial Trust Region in Nonlinear Programming," SIAM Journal on Scientific Computing, Vol. 18, No. 6, 1997, pp. 17881803.

[27] X. S. Zhang, Z. W. Chen and J. L. Zhang, "A Self-Adaptive Trust Region Method Unconstrained Optimization," Operations Research Transactions, Vol. 5, No. 1, 2001, pp. 53-62.

[28] N. I. M. Gould, D. Orban, A. Sartenaer and P. L. Toint, "Sensitivity of the Trust-Region Algorithms to Their Parameters," 4OR: A Quarterly Journal of Operations Research, Vol. 3, No. 3, 2005, pp. 227-241.

[29] K. Schittkowski, "More Test Examples for Nonlinear Programming Codes," Lecture Notes in Economics and Mathematical Systems, Springer Verlag, Berlin, Heideberg, Vol. 282, 1987.

[30] H. Y. Benson, "Nonlinear Optimization Models by AMPL: Cute Set." http://www.princeton.edu/ rvdb/ampl/nlmodels 